OPEN ACCESS

Edited by:

Dong Ho Park,

Kyungpook National University

Hospital, South Korea

Reviewed by:

Rahul Bhargava,

GS Medical College and

Hospital, India

Savleen Kaur,

Post Graduate Institute of Medical

Education and Research

(PGIMER), India

*Correspondence:

Mathias V. Melega

mvmelega@hotmail.com

Specialty section:

This article was submitted to

Ophthalmology,

a section of the journal

Frontiers in Medicine

Received: 26 April 2021 Accepted: 09 August 2021

Published: 27 August 2021

Citation:

Melega MV, dos Reis R, Lira RPC, de Oliveira DF, Arieta CEL and Alves $M$ (2021) Comparison Between Nylon and Polyglactin Sutures in Pediatric

Cataract Surgery: A Randomized Controlled Clinical Trial.

Front. Med. 8:700793.

doi: 10.3389/fmed.2021.700793

\section{Comparison Between Nylon and Polyglactin Sutures in Pediatric Cataract Surgery: A Randomized Controlled Clinical Trial}

\author{
Mathias V. Melega ${ }^{1 *}$, Roberto dos Reis ${ }^{1}$, Rodrigo Pessoa Cavalcanti Lira ${ }^{2}$, \\ Denise Fornazari de Oliveira ${ }^{1}$, Carlos Eduardo Leite Arieta ${ }^{1}$ and Monica Alves ${ }^{1}$ \\ ${ }^{1}$ School of Medical Sciences, University of Campinas, São Paulo, Brazil, ${ }^{2}$ School of Medical Sciences, Federal University of \\ Pernambuco (UFPE), Recife, Brazil
}

Purpose: To compare the performance of nylon sutures to that of polyglactin sutures in pediatric patients undergoing cataract surgery.

Setting: University of Campinas (UNICAMP), Campinas, São Paulo, Brazil

Design: A prospective, randomized, partially masked, single-site clinical trial. (https:// clinicaltrials.gov/ct2/show/NCT03812640).

Methods: A total of 80 eyes from 80 patients who underwent pediatric cataract surgery were randomized into two groups in block sizes of four. Group A consisted of 41 patients whose surgical incisions were sutured with polyglactin 10-0 material. Group B consisted of 39 patients whose surgical incisions were sutured with nylon 10-0 material. The primary outcome was frequency of suture-related complications in each group. Secondary outcomes were the frequency with which suture removal was necessary.

Results: The incidence of suture-related complications within 6 months of follow up was 0 out of 41 eyes (0.00\%) in the polyglactin group and 17 out of 39 eyes $(43.59 \%)$ in the nylon control group $(p<0.001)$. In all of the eyes with suture-related complications, the sutures were promptly removed. The most frequent complications were vascularization near the suture (17.95\%) and loose sutures (17.95\%). No ocular or systemic study-related adverse events were observed.

Conclusions: Polyglactin sutures were found to be safe and effective for pediatric patients undergoing cataract surgery. Their lower rate of complications and reduced likelihood of removal (and the subsequent need for general anesthesia) make their use preferrable to that of nylon sutures. This study represents the first controlled randomized clinical trial to compare nylon sutures to polyglactin sutures in pediatric patients undergoing cataract surgery.

Clinical Trial Registration: URL: https://clinicaltrials.gov/ct2/show/, Identifier: NCT03812640.

Keywords: pediatric cataract, suture, nylon, polyglactin, suture-related complications 


\section{INTRODUCTION}

Though rare, pediatric cataracts are a major cause of childhood blindness. Children deprived of adequate treatment experience worsened quality of life, and the socioeconomic costs are higher overall. The prevalence of pediatric cataracts is estimated to range from 1:10,000 to 4:10,000 in industrialized nations and from 5:10,000 to $15: 10,000$ in developing countries. Pediatric cataracts generate $\sim 200,000$ cases of blindness in children around the world each year as a result of unoperated cataracts, surgical complications, or consequences of diseases such as glaucoma and amblyopia (1-4).

Pediatric cataracts must be diagnosed as early as possible; a late diagnosis worsens the visual prognosis. Because the patients are children, trauma or eye rubbing in the postoperative period cannot be fully avoided. In addition, children's sclera exhibits limited rigidity, resulting in poor integrity of the surgical incision if no sutures are made. This situation requires surgeons to systematically suture the surgical incision in order to guarantee the perfect closure of the eyeball and prevent complications such as leakage of the aqueous humor with postoperative hypotonia, iris prolapse, or anterior synechiae formation, as well as to prevent intraocular infection caused by the entrance of microorganisms into the incision. In Brazil, this suture has been performed using 10-0 nylon for decades. Because nylon is a non-absorbable material, it can remain in the patient's cornea for years, predisposing the patient to suture loosening with the accumulation of mucus, corneal erosion, corneal neovascularization, infectious keratitis, (5-7) endophthalmitis, $(8,9)$ and giant papillary conjunctivitis $(10)$. Because of the risk of complications associated with suturing, the removal of these sutures is essentially obligatory, though they must be removed under sedation in a surgical facility $(11,12)$. Due to the need for nylon suture removal, other materials are being considered.

In 1998, a randomized clinical trial comparing polyglactin 10-0 to nylon 10-0 in adult cataract surgeries with a $5.2 \mathrm{~mm}$ incision demonstrated safety in incision closure and a low rate of complications associated with the use of polyglactin; because polyglactin can be absorbed by the body within 5670 days, additional interventions to remove it are not necessary $(13,14)$. In 2007 , a retrospective study on pediatric patients comparing absorbable polyglactin sutures to non-absorbable polyester sutures demonstrated a lower rate of complications in the patients who received the polyglactin sutures (15). This study found that the polyglactin sutures produced no complications and that their removal was not necessary; meanwhile, the polyester sutures were associated with complications in $18 \%$ of the cases in which they were used, and their removal was required. However, the study was retrospective and used vastly different group sizes.

To our knowledge, no controlled randomized clinical trials have been performed to compare sutures performed using nylon to those performed using polyglactin in pediatric patients. We performed the current study in an attempt to establish evidence-based conclusions regarding the prevention of suturerelated complications.
Our study is the first randomized clinical trial to compare nylon sutures to polyglactin sutures in terms of rates of complications and the frequency with which suture removal is necessary in pediatric patients undergoing cataract surgery.

\section{MATERIALS AND METHODS}

This study was a single-site, prospective, parallel-group, randomized, partially masked, phase 3 clinical trial. It was performed after approval from the University of Campinas research ethics committee and was conducted in accordance with the tenets of the Declaration of Helsinki and current legislation on clinical research. Written informed consent was obtained from all subjects after the explanation of the procedures and study requirements. The trial was registered and began in January 2019 (Comparison Between Nylon and Polyglactin Corneal Suture in Pediatric Cataract Surgery: A Randomized and Controlled Clinical Trial; ClinicalTrials.gov identifier: NCT03812640; https://clinicaltrials.gov/ct2/show/ NCT03812640). The inclusion criteria were patients 14 years of age or younger for whom pediatric cataract surgery was clinically indicated. The exclusion criteria were traumatic cataract with ocular perforation, cataract surgery associated with other procedures (such as glaucoma filtering surgery, vitreoretinal surgery, or corneal surgery), signs of ocular or periocular infection, advanced glaucoma, and severe ocular surface disease.

Data were collected from patients undergoing cataract surgery at the University of Campinas (UNICAMP) Clinical Hospital. Medical records, routine preoperative clinical exam data, data from intraoperative evaluations, and data from the 1st, $7 \mathrm{th}, 30 \mathrm{th}$, 90th, 120th, and 180th postoperative days were collected on each patient.

Patients were randomly divided into one of two groups approximately equal in size and stratified by age (0-6 months, 6-12 months, 1-3 years, 3-6 years, and older than 6 years of age). Group A had their surgical incisions sutured with polyglactin 100 material $\left(\right.$ Vicryl $^{\circledR}{ }^{\circledR}$, composed of polyglactin $910,10-0$ diameter, absorbable, a $0.62 \mathrm{~cm}, 3 / 8$ circle needle) at the end of cataract surgery. Patients in Group B had their surgical incisions sutured with nylon 10-0 material (composed of nylon monofilament, 100 diameter, absorbable, a $0.55 \mathrm{~cm}, 1 / 2$ circle needle) at the end of cataract surgery.

All cataract surgeries were performed by the same surgeon (M.V.M.) in accordance with the protocols used in the Department of Ophthalmology of the University of Campinas (UNICAMP). Preoperative antibiotics were not used. Briefly, the surgery protocol consisted of skin antisepsis with $10 \%$ povidoneiodine, placement of a sterile surgical drape to isolate lid margin and eyelashes, the application of four drops of $5 \%$ povidoneiodine into the conjunctival sac, and subsequent irrigation using a balanced salt solution. In cases of povidone-iodine allergy, an aqueous solution consisting of $0.05 \%$ chlorhexidine was used. Phacoaspiration using the Infiniti ${ }^{\circledR}$ phacoemulsifier (Alcon Laboratories Inc., Fort Worth, Texas, USA) was performed, and an AcrySof ${ }^{\circledR}$ MA60AC intraocular foldable lens (Alcon Laboratories Inc, Fort Worth, Texas, USA) was implanted in 
patients 6 months of age and older. Ultrasound energy was not used.

The Pediatric Cataract Department of the University of Campinas does not indicate intraocular lens implantation in patients younger than 6 months of age, and these patients remain aphakic. Intraocular lens implants are always recommended for patients 6 months of age and older. We chose to perform an a YAG laser posterior capsulotomy as an outpatient procedure; when patients were too young or otherwise unable to undergo the YAG procedure, we chose to perform a primary posterior capsulotomy (PPC) combined with an anterior vitrectomy (AV). With a few exceptions, patients 5 years of age and older exhibited the level of cooperation necessary to receive a YAG laser posterior capsulotomy.

We therefore performed one of three surgical combinations on each patient: on children younger than 6 months of age, we performed phacoaspiration combined with a PPC and an AV with no intraocular lens implant. In children 6 months of age and older who could not tolerate a YAG laser posterior capsulotomy, we performed phacoaspiration with an intraocular lens implant, as well as a PPC and an AV at the time of the cataract surgery. In children who were able to tolerate an outpatient YAG laser posterior capsulotomy, we performed phacoaspiration with an intraocular lens implant.

Clear corneal incision was used. Only one side port was made in all cases, and both incisions were sutured with the knots buried into the corneal side. The topical postoperative regimen consisted of $0.5 \%$ moxifloxacin combined with $0.1 \%$ dexamethasone every $3 \mathrm{~h}$ for 7 days only during waking hours. On the seventh postoperative day, this regimen was changed to only $0.1 \%$ dexamethasone, which was tapered over the course of 3 weeks.

The primary outcome was incidence of complications associated with sutures in each group. The suture-related complications were defined as corneal neovascularization close to the suture, loosening of the suture, accumulation of mucus on the suture, early suture rupture (within 2 weeks), aqueous humor leakage through the incision (as determined by the Seidel test), prolapse of the iris through the incision site, infectious or traumatic keratitis, endophthalmitis, and giant papillary conjunctivitis. In eyes with suture-related complications, the sutures were promptly removed. Secondary outcome was the need for suture removal under sedation in each group.

Sample size was calculated based on the frequency of suture-related complications described in the literature $(15,16)$ and using a two-tailed 95\% confidence interval, $80 \%$ power, an exposed/unexposed radius of 1 , and a null frequency of complications in the polyglactin suture group, which resulted in approximately 40 subjects per group. The trial would have been suspended if a difference between the two groups lower than a type I error $(\alpha)$ of $4 \%$ had been found in the preanalysis of 50,75 , and $100 \%$ of the patients included. Eligible patients were randomly assigned at a 1:1 ratio. Randomization was stratified by age in block sizes of four. One nurse generated the random allocation sequence using a computer randomization list, and another nurse enrolled and assigned the subjects to the interventions in a masked fashion. Sealed opaque envelopes were used for allocation and were opened immediately before surgery.

After the attribution of the interventions, the patients and their guardians were masked to the type of intervention. The surgeons were not masked because the type of suture used can easily be identified during surgery and in the biomicroscopy exam in the postoperative period.

\section{STATISTICAL ANALYSIS}

Continuous data have been expressed as the mean \pm standard deviation and range. Medians and interquartile ranges were used for variables with non-normal distribution. Betweengroup differences of continuous variables were compared using the Wilcoxon signed-rank test, and categorical variables were compared using the $x^{2}$ test or Fisher's exact test when appropriate. Multiple Firth logistic regressions were used to assess the effect of covariates on the studied outcomes. Analyses were performed using STATA 14.0 (StataCorp LP, College Station, TX, USA). Statistical significance was established when $p \leq 0.05$.

\section{RESULTS}

This study enrolled 80 patients between January 2019, and August 2020. These subjects were randomized into group A (polyglactin suture) or group B (nylon suture). In total, 41 patients in the polyglactin group and 39 patients in the nylon group completed the 6 months of follow-up care; no subjects were lost to follow up (Figure 1). Demographic data demonstrate homogeneity between the groups, as displayed in Table $\mathbf{1}$.

Table 2 shows the surgical options applied to each group.

Multiple logistic regression analysis was employed to determine the factors most closely associated with intraoperative complications, and the results are shown in Table 3.

Table 4 shows the results of the multiple logistic regression employed to assess the factors associated with suture complications and the need for suture removal.

\section{DISCUSSION}

Unlike in adult cataract surgery, pediatric cataract extraction surgery requires that the surgical incision be systematically sutured. In Brazil, the most commonly used suture material is currently nylon 10-0. Nylon is a monofilament and nonabsorbable material composed of polyamides that enables increased suture tension time and induces minimal cellular reactions. Vicryl is an absorbable synthetic compound made of polyglactin (a copolymer of glycolide and lactide) (14). Suture absorption occurs as a result of hydrolysis, which itself is generated by glycolic and lactic acids. Many nylon sutures must be removed from patients during the postoperative period. Because the patients in these cases are children, this normally simple removal procedure requires sedation. As was demonstrated in our patient groups (Table 1), there is a major association between pediatric cataract and systemic 


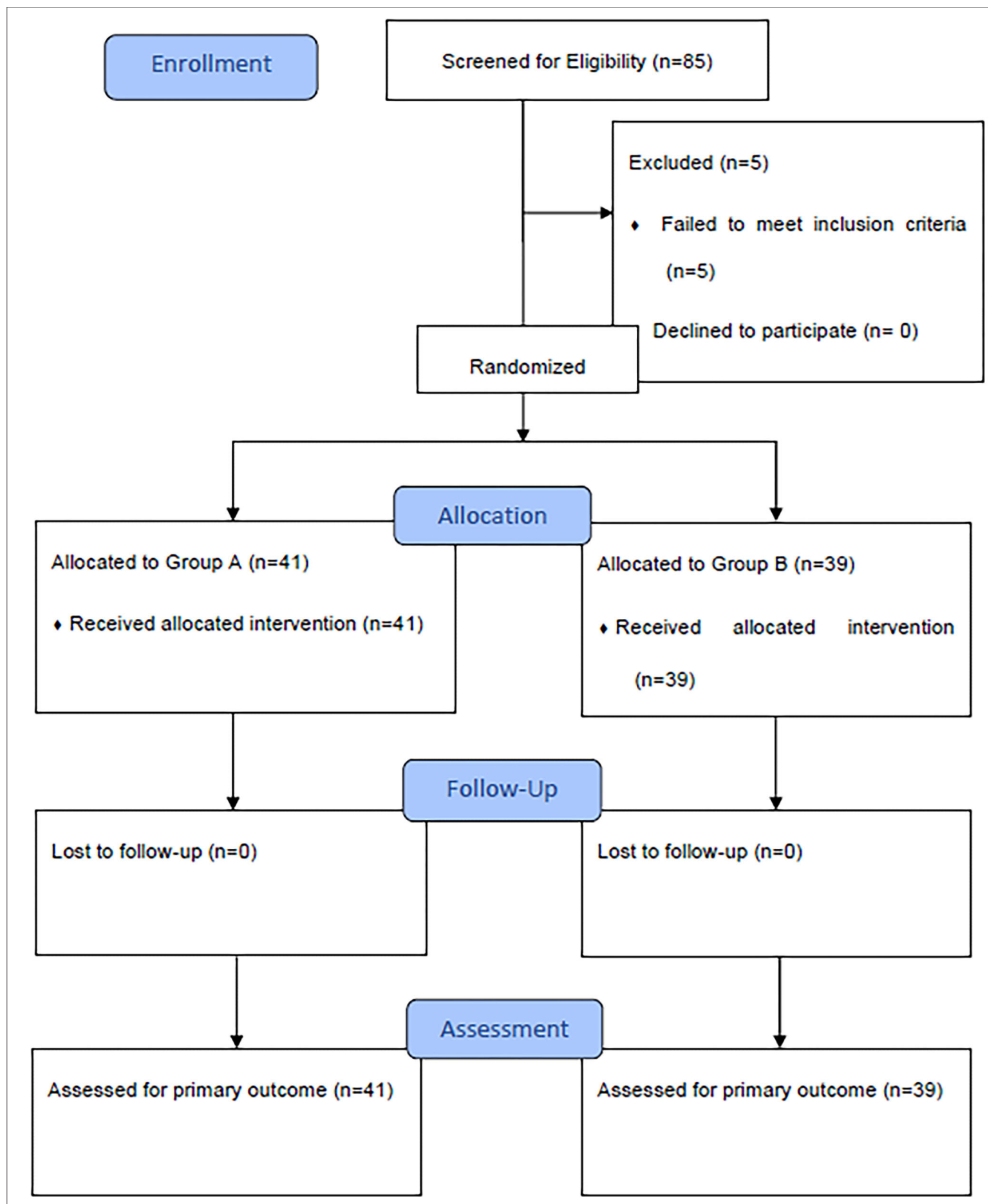

FIGURE 1 | Comparison Between nylon and polyglactin corneal sutures in pediatric cataract surgery: CONSORT flow diagram. 
TABLE 1 | Demographic characteristics of pediatric cataract patients in the polyglactin suture group and the nylon suture group.

\begin{tabular}{|c|c|c|c|}
\hline & $\begin{array}{l}\text { Polyglactin } \\
\text { group }\end{array}$ & Nylon group & $p$-Value \\
\hline Sex, $N(\%)$ Male & $21(51.22)$ & $26(66.67)$ & $0.161^{*}$ \\
\hline $\begin{array}{l}\text { Age (months), mean } \\
\pm \text { SD (median; 25\% } \\
\text { percentile; } 75 \% \\
\text { percentile) }\end{array}$ & $\begin{array}{c}45.76 \pm 50.24 \\
(16.00 ; 7.00 \\
72.00)\end{array}$ & $\begin{array}{c}42.49 \pm 46.41 \\
(24.00 ; 5.00 \\
72.00)\end{array}$ & $0.780^{\dagger}$ \\
\hline $\begin{array}{l}\text { Operated eye, } N(\%) \\
\text { OD }\end{array}$ & $18(43.90)$ & $25(64.10)$ & $0.070^{*}$ \\
\hline $\begin{array}{l}\text { Systemic } \\
\text { comorbidities, } N(\%)\end{array}$ & & & $0.151^{*}$ \\
\hline Marfan syndrome & $3(7.32)$ & $7(17.95)$ & \\
\hline Toxoplasmosis & $1(2.44)$ & $1(2.56)$ & \\
\hline Ichthyosis & $1(2.44)$ & $1(2.56)$ & \\
\hline Galactosemia & $0(0.00)$ & $3(7.69)$ & \\
\hline Down syndrome & $1(2.44)$ & $2(5.13)$ & \\
\hline Cockayne syndrome & $0(0.00)$ & $1(2.56)$ & \\
\hline No comorbidities & 35 (85.37) & $24(61.54)$ & \\
\hline $\begin{array}{l}\text { Ocular } \\
\text { comorbidities, N (\%) }\end{array}$ & & & $0.548 \#$ \\
\hline Chorioretinitis scarring & $1(2.44)$ & $0(0.00)$ & \\
\hline $\begin{array}{l}\text { Persistent fetal } \\
\text { vasculature }\end{array}$ & $1(2.44)$ & $0(0.00)$ & \\
\hline Congenital glaucoma & $1(2.44)$ & $1(2.56)$ & \\
\hline Strabismus & $1(2.44)$ & $0(0.00)$ & \\
\hline Traumatic cataracts & $0(0.00)$ & $1(2.56)$ & \\
\hline Aniridia & $1(2.44)$ & $0(0.00)$ & \\
\hline No comorbidities & $36(87.80)$ & 37 (94.87) & \\
\hline
\end{tabular}

SD, Standard Deviation; OD, Right Eye.

${ }^{\star}$ Chi-Squared test.

${ }^{\dagger}$ Wilcoxon signed-rank test.

\#Fisher's exact test.

diseases (Marfan syndrome, Down syndrome, congenital cardiac abnormalities, ichthyosis, inborn errors of metabolism, toxoplasmosis, rubella, syphilis, and other congenital infections). These comorbidities may increase the rate of complications in cases requiring general anesthesia in a group of patients whose age already makes them more vulnerable in this procedure $(17,18)$.

In addition to the risks associated with general anesthesia, suture removal itself also increases the risk of endophthalmitis in that it makes it easier for microorganisms to enter the eye as the string is being pulled (8). Certain measures must be taken to prevent this complication, including the use of $5 \%$ povidoneiodine before the procedure, cutting the suture at one end to prevent the external portion of the string from entering the eye, postoperative topical antibiotics, and outpatient follow-up care to catch and treat any infections as early as possible.

Other researchers have also compared different suture materials for use in pediatric cataract surgery. Bar-sela et al. (15). performed a retrospective study to compare polyester 100 (Mersilene $\AA$ ) to polyglactin 10-0 (Vicryl $\AA$ ) over 6 months of follow up and, similar to our results, found no suture-related
TABLE 2 | Pediatric cataract surgery outcomes organized by suture material used (polyglactin vs. nylon sutures).

\begin{tabular}{|c|c|c|c|}
\hline & Polyglactin group & Nylon group & $p$-Value \\
\hline & $\boldsymbol{N}(\%)$ & $\boldsymbol{N}(\%)$ & \\
\hline Surgery type & & & $0.776^{*}$ \\
\hline Phaco + PPC + AV & $23(56.10)$ & $25(64.10)$ & \\
\hline Phaco + PPC + AV + IOL & $7(17.07)$ & $6(15.38)$ & \\
\hline Phaco + IOL & $11(26.83)$ & $8(20.51)$ & \\
\hline Intraoperative complications & & & 0.999\# \\
\hline PCR & $1(2.44)$ & $1(2.56)$ & \\
\hline Iris damage & $1(2.44)$ & $1(2.56)$ & \\
\hline None & 39 (95.12) & 37 (94.87) & \\
\hline Suture-related complications & & & $<0.001 \#$ \\
\hline Vascularized suture & $0(0.00)$ & $7(17.95)$ & \\
\hline Suture loosening & $0(0.00)$ & 7 (17.95) & \\
\hline Suture with mucus & $0(0.00)$ & $1(2.56)$ & \\
\hline Suture rupture & $0(0.00)$ & $2(5.13)$ & \\
\hline No complications & 41 (100.00) & $22(56.41)$ & \\
\hline
\end{tabular}

PPC, primary posterior capsulotomy; AV, anterior vitrectomy; IOL, intraocular lens; Phaco, phacoaspiration; $P C R$, posterior capsule rupture.

${ }^{*}$ Chi-Squared test.

\#Fisher's exact test.

TABLE 3 | Multiple logistic regression analysis applied to data on intraoperative complications in pediatric cataract surgeries in which either nylon or polyglactin sutures were used.

\begin{tabular}{lcc}
\hline & Odds ratio (95\% Cl) & $p$-Value \\
\hline Sex & Reference & 0.178 \\
Male & $7.43(0.40-137.25)$ & \\
Female & $1.02(0.99-1.04)$ & 0.062 \\
Age (months) & & 0.232 \\
Systemic comorbidities & Reference & \\
No & $0.21(0.02-2.69)$ & \\
Yes & & 0.820 \\
Ocular comorbidities & Reference & \\
No & $1.44(0.06-32.22)$ & - \\
Yes & & \\
Surgery type & Reference & \\
Phaco + PPC + AV & $0.43(0.02-10.86)$ & 0.606 \\
Phaco + PPC + AV + IOL & $0.55(0.07-4.54)$ & 0.578 \\
Phaco + IOL & & - \\
Type of suture material & Reference & \\
Nylon & $2.04(0.25-16.63)$ & 0.504 \\
Polyglactin &
\end{tabular}

PPC, primary posterior capsulotomy; $A V$, anterior vitrectomy; IOL, intraocular lens; Phaco, phacoaspiration; $\mathrm{Cl}$, confidence interval.

complications in the polyglactin 10-0 group. Matalia et al. performed a non-randomized prospective study to compare the same suture materials considered herein in pediatric cataract surgeries and obtained similar results (16). As in the surgeries performed herein, these researchers found the polyglactin string to be more difficult to work with and bury. In Matalia et al. 
TABLE 4 | Multiple logistic regression analysis applied to suture complications and the need for suture removal in cases of pediatric cataract surgeries with either nylon or polyglactin sutures.

\begin{tabular}{|c|c|c|}
\hline & Coefficient (95\% Cl) & $p$-Value \\
\hline \multicolumn{3}{|l|}{ Sex } \\
\hline Male & Reference & \\
\hline Female & $1.61(0.38-6.86)$ & 0.519 \\
\hline Age (months) & $1.01(0.99-1.03)$ & 0.334 \\
\hline \multicolumn{3}{|l|}{ Systemic comorbidities } \\
\hline No & Reference & \\
\hline Yes & $1.08(0.23-5.17)$ & 0.922 \\
\hline \multicolumn{3}{|l|}{ Ocular comorbidities } \\
\hline No & Reference & \\
\hline Yes & 4.85 (0.29-81.94) & 0.273 \\
\hline \multicolumn{3}{|l|}{ Surgery type } \\
\hline Phaco + PPC + AV & Reference & \\
\hline Phaco $+\mathrm{PPC}+\mathrm{AV}+\mathrm{IOL}$ & $0.43(0.05-3.54)$ & 0.437 \\
\hline Phaco + IOL & $0.13(0.01-1.90)$ & 0.136 \\
\hline \multicolumn{3}{|c|}{ Intraoperative complications } \\
\hline No & Reference & \\
\hline Yes & $1.64(0.21-12.86)$ & 0.636 \\
\hline \multicolumn{3}{|l|}{ Type of suture material } \\
\hline Nylon & Reference & \\
\hline Polyglactin & 61.69 (3.43-1110.93) & 0.005 \\
\hline
\end{tabular}

PPC, primary posterior capsulotomy; AV, anterior vitrectomy; IOL, intraocular lens; Phaco, phacoaspiration; $\mathrm{Cl}$, confidence interval.

and in our study, this finding is merely anecdotal and was not measured quantitatively.

Sukhija and Kaur (20) performed a non-randomized prospective study to compare the efficacy and outcome of viscosealing the incisions using $1.4 \%$ sodium hyaluronate with polyglactin sutures in children $<5$ years. They concluded viscosealing is comparable to suturing of incisions in children undergoing cataract surgery.

Bartholomew et al. (19) also performed a prospective randomized study to compare polyglactin $8-0$, nylon $10-0$, and silk 8-0, but they reported a greater risk of complications in the polyglactin group within the first month after surgery relative to their other study groups. This finding differs from more recent studies because, according to the authors, they experienced difficulty in knotting the polyglactin string, which resulted in ineffective incision closure. It is important to note that their study was performed in 1976, before the advent of phacoemulsification; therefore, the number of sutures required to close the incision was considerably higher than is required today. This large number of sutures predisposed patients to experiencing suturerelated complications. Furthermore, this study was performed on adult patients.

As in other well-known studies on pediatric patients $(15,16)$,

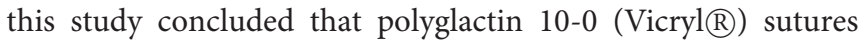
were associated with fewer complications than polyester 100 (Mersilene $(\mathrm{R})$ ) sutures; the results therefore demonstrate the benefits of this polyglactin product. Our study seems to be the first to compare different suture materials in prospective and randomized groups of children. We found that the only factor associated with suture-related complications in and following pediatric cataract surgery was the type of material used: subjects who received the nylon sutures were 61.7 times more likely to experience suture-related complications and to require suture removal relative to subjects who received polyglactin sutures $(\mathrm{OR}=61.69 ; 95 \% \mathrm{CI}: 3.43-1110.93 ; p=0.005)$. The most frequent complications were vascularization near the suture (17.95\%) and suture loosening (17.95\%). In the nylon group, the mean time for suture removal was $88.23 \pm 59.50$ days (median $=60$ days). It is important to note that there were no significant differences between the groups in terms of types of surgery $(p=0.776)$ or intraoperative complications ( $p$ $=0.776$ ).

This study presents some limitations. The degree of astigmatism induced was not assessed due to the lack of adequate methods available for measurement in children, who are unable to undergo in-office keratometry. The exact amount of time required for polyglactin absorption was not determined; however, at the end of the 6-month follow-up period, the biomicroscope exams performed on the polyglactin group revealed no evidence of any suture remains. Our study would have been improved by data comparing the average amount of time spent on suturing in each of the groups, since, as mentioned previously, we experienced greater difficulty and required more time to suture with polyglactin string than with nylon. In a future study, we plan to assess the cost-effectiveness of the use of polyglactin string: though this material is more expensive than nylon, it clearly reduces the need for both additional procedures and the use of general anesthesia for suture removal.

Our study is the first randomized clinical trial to demonstrate that absorbable polyglactin 10-0 sutures are safe for use in pediatric cataract surgery and result in fewer postoperative complications than non-absorbable nylon 10-0 sutures. None of the patients who received polyglactin sutures required suture removal under sedation; these patients were therefore spared the risks associated with general anesthesia and with suture removal itself.

\section{DATA AVAILABILITY STATEMENT}

The raw data supporting the conclusions of this article will be made available by the authors, without undue reservation.

\section{ETHICS STATEMENT}

The studies involving human participants were reviewed and approved by Ethics committee of the School of Medical Sciences, University of Campinas (UNICAMP). Written informed consent to participate in this study was provided by the participants' legal guardian/next of kin.

\section{AUTHOR CONTRIBUTIONS}

MM, RL, CA, and MA contributed to conception and design of the study. MM and RR organized the database. MM, RR, 
DO, and MA performed the statistical analysis. MM and MA wrote the first draft of the manuscript. MM, RR, RL, DO, $\mathrm{CA}$, and MA wrote sections of the manuscript. All authors contributed to manuscript revision, read, and approved the submitted version.

\section{FUNDING}

Financial support: São Paulo Research Foundation (FAPESP) Grant No. 2014/19138-5 and School of Medical Sciences, University of Campinas (UNICAMP).

\section{REFERENCES}

1. Foster A, Gilbert C, Rahi J. Epidemiology of cataract in childhood: a global perspective. J Cataract Refract Surg [Internet]. (1997) 23:601-4. doi: 10.1016/S0886-3350(97)80040-5

2. Gilbert CE, Wood M, Waddel K, Foster A. Causes of childhood blindness in east Africa: results in 491 pupils attending 17 schools for the blind in Malawi. Kenya and Uganda. Ophthalmic Epidemiol [Internet]. (1995) 2:7784. doi: 10.3109/09286589509057086

3. Haargaard B, Wohlfahrt J, Fledelius HC, Rosenberg T, Melbye M. Incidence and Cumulative Risk of Childhood Cataract in a Cohort of 2.6 Million Danish Children. Invest Ophthalmol Vis Sci [Internet]. (2004) 45:131620. doi: 10.1167/iovs.03-0635

4. WHO | Priority eye diseases [Internet]. WHO. World Health Organization. (2018). Available online at: http://www.who.int/blindness/causes/priority/en/ index1.html

5. Wilson BME. Pediatric Cataracts : Overview Classification (Categorization). (2016). Available online at: https://www.aao.org/disease-review/pediatriccataracts-overview

6. Heaven CJ, Boase DL. Suppurative keratitis with endophthalmitis due to biodegraded full thickness monofilament nylon corneal sutures. Eur J Implant Refract Surg [Internet]. (1993) 5:164-8. doi: 10.1016/S0955-3681(13) 80436-4

7. Lee BJ, Smith SD, Jeng BH. Suture-related corneal infections after clear corneal cataract surgery. J Cataract Refract Surg [Internet]. (2009) 35:93942. doi: 10.1016/j.jcrs.2008.10.061

8. Culbert RB, Devenyi RG. Bacterial endophthalmitis after suture removal. $J$ Cataract Refract Surg. (1999) 25:725-7. doi: 10.1016/S0886-3350(99)00020-6

9. Khurshid GS, Fahy GT. Endophthalmitis secondary to corneal sutures: Series of delayed-onset keratitis requiring intravitreal antibiotics. J Cataract Refract Surg. (2003) 29:1370-2. doi: 10.1016/S0886-3350(03)00404-8

10. Acheson CF, London L. Ocular Morbidity Due to Monofilament Nylon Corneal Sutures. Eye [Internet]. (1991) 5:106-12. doi: 10.1038/eye.1991.20

11. Danjoux JP, Reck AC. Corneal sutures: Is Routine Removal Really Necessary? Eye (Lond). 8:339-42. doi: 10.1038/eye.1994.70

12. Jackson H, Bosanquet R. Should nylon corneal sutures be routinely removed? Br J Ophthalmol. (1991) 75:663-4. doi: 10.1136/bjo.75.11.663

13. Bainbridge JWB, Teimory M, Kirwan JF, Rostron CK, A. prospective controlled study of a $10 / 0$ absorbablepolyglactin suture for corneal

\section{ACKNOWLEDGMENTS}

We would like to thank our colleagues who greatly assisted in this research: Fabrício Ferreira do Santos provided assistance with statistics. Arthur Fernandes provided assistance with statistics. Danielle Deremo Cosimo provided English-language editing.

\section{SUPPLEMENTARY MATERIAL}

The Supplementary Material for this article can be found online at: https://www.frontiersin.org/articles/10.3389/fmed. 2021.700793/full\#supplementary-material

incision phacoemulsification. Eye. (1998) 12:399-402. doi: 10.1038/eye.19 98.94

14. Ethicon. Ethicon product catalog. (2014) p. 208-30. Available online at: http:// www.ecatalog.ethicon.com/sites/default/files/ethicon-catalog.pdf

15. Bar-Sela SM, Spierer O, Spierer A. Suture-related complications after congenital cataract surgery: Vicryl versus Mersilene sutures. J Cataract Refract Surg. (2007) 33:301-4. doi: 10.1016/j.jcrs.2006.10.039

16. Matalia J, Panmand P, Ghalla P. Original article comparative analysis of nonabsorbable 10 - 0 nylon sutures with absorbable 10 - 0 Vicryl sutures in pediatric cataract surgery. (2018) 661-4. doi: 10.4103/ijo.IJO_654_17

17. Thomas FA. Complications following general anesthesia. Curr Res Anesth Analg. (1940) 19:94-6.

18. Benezra D. The surgical approaches to paediatric cataract. Eur J Implant Refract Surg [Internet]. (1990) 2:241-4. doi: 10.1016/S0955-3681(13)80091-3

19. Bartholomew RS, Phillips CI, Munton CG. Vicryl (polyglactin 910) in cataract surgery. A controlled trial. Br J Ophthalmol. (1976) 60:536-8. doi: 10.1136/bjo.60.7.536

20. Sukhija J, Kaur S. Comparison of two methods of wound closure in paediatric cataract surgery. J Clin Exp Ophthalmol. (2018) 9:766. doi: $10.4172 / 2155-9570.1000766$

Conflict of Interest: The authors declare that the research was conducted in the absence of any commercial or financial relationships that could be construed as a potential conflict of interest.

Publisher's Note: All claims expressed in this article are solely those of the authors and do not necessarily represent those of their affiliated organizations, or those of the publisher, the editors and the reviewers. Any product that may be evaluated in this article, or claim that may be made by its manufacturer, is not guaranteed or endorsed by the publisher.

Copyright (C) 2021 Melega, dos Reis, Lira, de Oliveira, Arieta and Alves. This is an open-access article distributed under the terms of the Creative Commons Attribution License (CC BY). The use, distribution or reproduction in other forums is permitted, provided the original author(s) and the copyright owner(s) are credited and that the original publication in this journal is cited, in accordance with accepted academic practice. No use, distribution or reproduction is permitted which does not comply with these terms. 